\title{
BMJ Open 'It would not be tolerated in any other profession except medicine': survey reporting on undergraduates' exposure to bullying and harassment in their first placement year
}

\author{
Anja Timm
}

To cite: Timm A. 'It would not be tolerated in any other profession except medicine': survey reporting on undergraduates' exposure to bullying and harassment in their first placement year. BMJ Open 2014;4:e005140. doi:10.1136/bmjopen-2014005140

- Prepublication history for this paper is available online. To view these files please visit the journal online (http://dx.doi.org/10.1136/ bmjopen-2014-005140).

Received 4 March 2014 Revised 16 June 2014 Accepted 17 June 2014

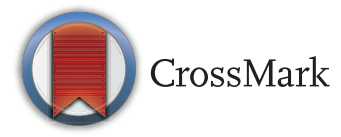

Academic Unit of Medical Education/Medical Education Development Unit, Faculty of Medicine, University of Southampton, Southampton, UK

Correspondence to Dr Anja Timm;

A.Timm@soton.ac.uk

\section{ABSTRACT}

Objectives: To determine the extent to which undergraduate medical students experience (and/or witness) bullying and harassment during their first year on full-time placements and to compare with new General Medical Council (GMC) evidence on bullying and harassment of doctors in training.

Setting: A UK university offering medical and nursing undergraduate programmes.

Participants: 309 medical and nursing undergraduate students with 30-33 weeks' placement experience (123 medical students and 186 nursing students); overall response rate: $47 \%$.

Primary and secondary outcome measures: $(A)$ students' experience of bullying and harassment; (B) witnessing bullying and harassment; (C) actions taken by students; (D) comparison of medical and nursing students' data.

Results: Within 8 months of starting clinical placements, a fifth of medical and a quarter of nursing students reported experiencing bullying and harassment. Cohorts differ in the type of exposure reported and in their responses. Whereas some nursing students follow incidences with query and challenge, most medical students acquiesce.

Conclusions: Bullying and harassment of medical (and nursing) students-as well as witnessing of such incidents-occurs as soon as students enter the clinical environment. This augments evidence published by the GMC in its first report on undermining of doctors in training (December 2013). The data suggest differences between nursing and medical students in how they respond to such incidents.

\section{BACKGROUND}

In 2012 the General Medical Council (GMC) introduced a new indicator entitled 'undermining' in its annual National Training Survey (NTS). ${ }^{1}$ Their first dedicated report on undermining showed that $13 \%$ of respondents reported having been victims of

\section{Strengths and limitations of this study}

First published UK survey on bullying and harassment of undergraduate medical students.

- Unique comparison of undergraduate medical and nursing students.

- Findings closely match the General Medical Council's report on undermining of doctors in training (2013) and add to the debate on bullying and harassment raised by Francis Report (2012).

- Discussion highlights the need to integrate policy on bullying and harassment within undergraduate medical education if bullying is to be addressed effectively.

- Indicative rather than conclusive insights into medical student mistreatment. Providing the first published UK data of its' kind, it is necessarily a single centre study only. The sample size is small; however, a good response rate of $47 \%$ was achieved.

bullying and harassment in their training post and some $20 \%$ had witnessed someone else being bullied. ${ }^{2}$ The Royal College of Obstetricians and Gynaecologists issued a joint statement with the Royal College of Midwives condemning such practices, ${ }^{3}$ as did the Association of Surgeons in Training. ${ }^{4}$ The GMC proposed that they would follow-up reported instances of bullying and carry to a series of targeted check visits to investigate how concerns were being responded to locally. ${ }^{2}$

That bullying and harassment of doctors in training occurs is well known. According to Quine's (2002) UK workplace survey $37 \%$ of junior doctors reported having been bullied in the past year and this did not vary by grade or age. ${ }^{5}$ Paice's ${ }^{6}$ survey showed $18 \%$ of trainees had been exposed to bullying and identified senior doctors as the main source of this, but also noted that only a third 
(32\%) reported the incident. Those who opted not to report either deemed the matter insufficiently serious $(31 \%)$ or were afraid of the consequences $(25 \%)$.

US surveys on medical students' experiences of bullying and harassment are plentiful, with an important comparison of data from 16 medical schools published in 2006. ${ }^{7}$ Until now no quantitative data charting UK medical students' experiences of bullying has been published although valuable qualitative studies have highlighted problematic aspects of students' socialisation into medicine and revealed the 'hidden curriculum, ${ }^{8-10}$ which Hafferty describes as 'a set of influences that function at the level of organisational structure and culture'. ${ }^{11}$ Elsewhere research has revealed the professionalism dilemmas for students that result from poor role-modelling, ${ }^{12}{ }^{13}$ and the negative impact on students' confidence in clinical skills. ${ }^{14}$ International research confirms this rather bleak picture. ${ }^{15} 16$

This survey provides the first quantitative indicator of bullying experienced by current undergraduate medical students at a British medical school. Unlike previous research this includes initial full-time placements, which commonly occur in year 3 of UK undergraduate medical programmes. The analysis here also offers unique comparative data gathered from nursing students who were on placement at the same hospital sites as the medical students. This paper describes how medical student respondents reacted to their exposure to bullying and harassment and reveals considerable differences in how nursing and medical students respond to these challenges.

For orientation purposes box 1 provides a brief summary of how undergraduate programmes in medicine and nursing at the research site (a University in England) compare in terms of initial full-time placement year.

\section{METHODS}

An online questionnaire was developed, piloted and ethics approval was granted by the Faculty of Medicine Ethics Committee in March 2012 (Ref: SOMSEC 085.10). The survey was open online for 6 weeks between April-May 2012. As the survey went live, the participating medical and nursing students had spent

Box 1 Comparison of the initial full-time placements in medicine and nursing at the research site (a University in England)

Medical students' first full-time placements start in year 3. Per week they spend 4 days on placement and 1 day attending lectures. Small groups (6-8 students: 1 clinical teacher) rotate through specialties and subspecialties. Early patient contact (not covered by the survey) happens throughout years 1 and 2 on a weekly basis.

- Nursing students' placements start in year 1, when they are expected to work shifts alongside their dedicated mentors $(1: 1)$; the majority of students will undertake placements lasting 10 weeks each. between 33 and 30 weeks on full-time placements, respectively; medical students were in year 3 and nursing students in year 2 of their degrees.

Recruitment: Students on both cohorts received an initial email invitation, followed by weekly reminders from administrative and/or academic staff while the survey was live (ie, at least six times). Additional awareness raising initiatives included public display screen reminders and 'shout outs' at lectures. Finally, members of the student reference group and the research team distributed flyers at curricular and extracurricular events.

The questionnaire had a total of 18 questions; only those relevant to bullying and harassment are reported here $(\mathrm{Q} 8-\mathrm{Q} 10)$ :

\begin{abstract}
Q8: “Thinking about all your placements, have you witnessed or experienced any incidents of bullying or harassment either towards yourself or other people? (bullying or harassment refers to something happening which is unwelcome, unwarranted and causes a detrimental effect)." Respondents were asked to respond: No/Yes/ Prefer not to answer.
\end{abstract}

Q9: "If yes, can you provide brief details?", followed by free text box.

Q10: "What did you do in this situation?", followed by free text box.

A total of 309 completed online questionnaires were collected from a population of 653 students. The data were imported from the online survey tool into Excel. Where respondents had provided multiple answers, although only one answer was eligible, all of the multiple answers were removed for that question. This report provides a descriptive analysis of the remaining data. Frequencies were examined within Excel (no statistical tests were performed). Data entered through the open text format were read repeatedly and grouped as presented below. Some minor editing of free text comments was undertaken to increase legibility, for example, consistent capitalisation was applied and spelling mistakes were corrected. Where specific locations were mentioned, these were removed.

\section{RESULTS}

As shown in table 1 below, there was some difference in the response rate between the nursing and medical student cohorts.

Table 1 Details of sample, respondents ( $\mathrm{n}$ ) and response rate per cohort

\begin{tabular}{llll}
\hline Programme & $\begin{array}{l}\text { Student } \\
\text { population }\end{array}$ & $\begin{array}{l}\text { Number of } \\
\text { respondents }\end{array}$ & $\begin{array}{l}\text { Response } \\
\text { rate (\%) }\end{array}$ \\
\hline Medicine & 271 in year 3 & 123 & 45 \\
Nursing & 382 in year 2 & 186 & 49 \\
\hline
\end{tabular}


Figure 1 Responses to 'Have you witnessed or experienced any incidents of bullying or harassment either towards yourself or other people?' (in percentages).

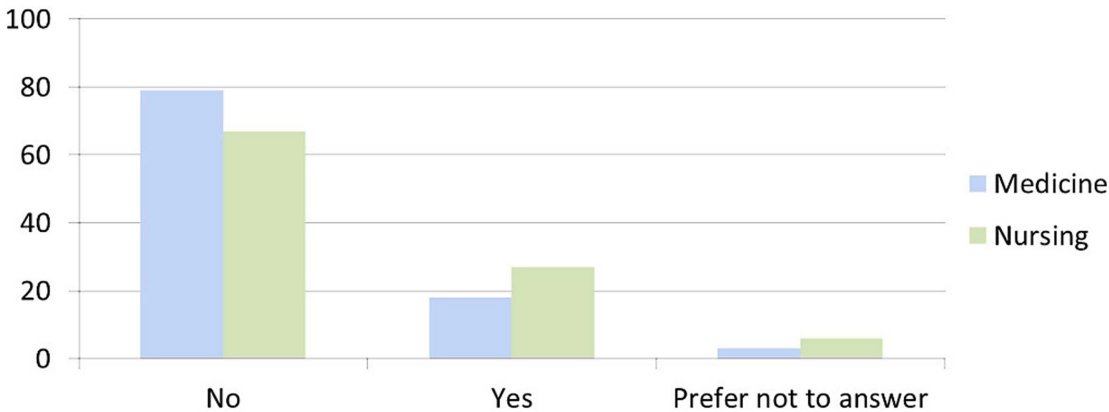

Experiencing and witnessing bullying and harassment (prevalence)

Respondents were asked: 'Thinking about all your placements, have you witnessed or experienced any incidents of bullying or harassment either towards yourself or other people? (bullying or harassment refers to something happening which is unwelcome, unwarranted and causes a detrimental effect).' The answer choices were: yes/no/prefer not to answer (figure 1).

Nursing students were more likely to report exposure: $67 \%$ responded 'no' and 27\% responded 'yes'. From the medical student cohort, $79 \%$ responded 'no' and $18 \%$ responded 'yes'. Six per cent of nursing respondents chose the option 'prefer not to answer', compared with $3 \%$ from medicine.

\section{Details of what happens and how students react}

Respondents were asked 'If yes, can you provide brief details?' and 'What did you do in this situation?'; for both of these questions, the answer format was free text. From the total number of survey respondents (309) there were a total of 74 open text responses (24\%). Of the open text comments, 21 comments were submitted by medical students $(28 \%)$ and 53 comments were provided by nursing students $(72 \%)$. The responses were categorised by the author into: personal experiences/witness reports/experiences of 'bad atmosphere' and incidents involving patients and examples of each are provided below.

In their responses, nursing respondents frequently refer to their 'mentor' and it should be noted that this is the term used locally to describe the registered nurse who is their dedicated clinical teacher while they are on placement. Throughout, the results tables the quotes are colour coded: responses from medical students are on a blue background and those from nursing students are on a green background. In addition, each quote is given a number, which starts with either $\mathrm{M}$ (=medicine) or $\mathrm{N}$ (=nursing) to denote the profession.

1. The category with the most responses $(43 \%)$ details participants' reports of their own experiences of bullying and harassment (table 2), in total, it was

Table 2 Responses from nursing and medical students detailing their own experiences of bullying and harassment (these are direct quotes selected by the author to indicate the spread and issues involved)

\section{'If yes, can you provide brief details?'}

M3 More talking down, derogatory comments to students than outright bullying

M15 One consultant in particular was rude and obtuse on more than one occasion and incredibly condescending. His expectations were very unrealistic and he was impatient with us. This did not stop me getting an A grade overall from my second consultant

M19 Being told by a consultant that I 'didn't know anything'

N9 There have been issues where I have been ridiculed during placement, felt unwanted, not part of the team and also intimidated by members of staff, I felt hindered because I was not able to initiate any tasks

N29 Was told men should not be nurses. And witnessed both sexual innuendo being aimed at students by both staff and patients

N45 Certain member of staff making me and other members not feel valued and not wanted to teach. This knocked my confidence due to their attitude towards me

\section{'What did you do in this situation?'}

There's not much you can do other than note it on feedback forms

Spent more time with my second consultant after what seemed like an argument without resolve was going to take place

Ignored the comment and consequently avoided said consultant

Confronting the staff member in question in a professional manner and liaising with my mentor about this incident to ensure that the issue could be resolved

Reported the matter and supported the individual

I avoided the situation until I had a chance to talk with her the next day 
reported 32 times, with 10 responses from medical students and 22 responses from nursing students.

2. The second most observed category among the open text comments $(27 \%)$ relates to participants' witness accounts of bullying and harassment (table 3 ). There were a total of 20 responses with 6 from medical students and 14 from nursing students.

3. Respondents reported experiencing a 'bad atmosphere' on 12 occasions in the open text comments $(16 \%)$. This was reported by four medical students and eight nursing students, with examples provided below (table 4).

4. The final category relates to reported incidents of bullying and harassment involving patients $(14 \%$ of the open text comments received). It includes one report from a medical student and nine from nursing students (table 5).

\section{DISCUSSION}

Our survey findings concur with prior research, which indicates that healthcare students on clinical placements continue to be exposed to bullying and harassment. Additionally, it highlights just how early on such incidences occur in medical as well as nursing education and how unsettling they are to students on initial fulltime placements. From the very beginning of clinical placements, UK medical and nursing students are exposed to highly problematic role-modelling.

\section{Differences between the cohorts}

Our survey findings indicate that prevalence and types of exposure to bullying and harassment differ between medical and nursing students. First, prevalence is higher in nursing. However, open text comments by nursing students include a level of reflection about the nature and dynamics of teams or the likely reason why bullying behaviours occur that are largely absent from the medical student's accounts. This suggests that nursing students may be more able to reflect on their experiences, which in turn may relate to patterns of recruitment, and/or the support available to them in the ward environment. In particular, the nursing students-unlike the medical students-worked closely (usually 1:1) with a dedicated clinical teacher (their 'mentor'), which might make a crucial difference to their contextual understanding and in backing them through their first steps on the wards.

The percentage of medical student respondents who experienced (or witnessed) bullying and harassment in our survey is identical to that reported by junior doctors in $2004,{ }^{6}$ and within $2 \%$ of that reported by the GMC in $2013 .^{2}$ While this provides some reassurance about the trustworthiness of our data, it is nevertheless shocking that students report exposure so early on. It should also be borne in mind that according to a British Medical Association report doctors are 'less likely to admit to experiencing bullying and harassment compared to other healthcare workers'. ${ }^{17}$

Table 3 Responses from nursing and medical students providing witness accounts of bullying and harassment in the clinical environment (these are direct quotes selected by the author to indicate the spread and issues involved)

\section{'If yes, can you provide brief details?'}

M8 In my very first placement a registrar made an FY1 cry on the ward round by deliberately making her feel embarrassed in front of a patient for a very minor (and easily made) error

M11 Particularly on (...) surgical placement (...). The consultant had not taught us anything and towards the end of the placement decided to test our knowledge by throwing questions at us. The fact that it was on the spot and that we couldn't necessarily remember all the details correctly he decided to make us feel completely inadequate and not constructive at all

N18 Another student was being intimidated by her mentor

N35 New students (year 1), ie, placements $1 / 2$, [are] prone to bullying in terms of being made errand runners and tea makers with little interest in us for teaching as staff feel we know too little to be of any help during a shift often felt this due to snide remarks. Often felt like a burden on shift as changes in Trust was causing staff anxiety. Felt we had to work extra hard even past own limitations to prove worthiness sometimes

\section{'What did you do in this situation?'}

I comforted the FY1 and took over the organisational bits of the ward round (such as making sure obs charts and notes were available on demand)

I felt really belittled and didn't feel like doing anything to be honest

I spoke with my mentor who explained a complaint should be made to the university and to the ward sister Ward Managers were great!!! Often protecting us by reading out at staff meetings the terms of our supernumerary status and obligation of staff to teach us (NMC code etc). Often offering us time to debrief/reflect on experiences on ward and problem solve together if we had any issues. Worked hard and never declined any task I felt in my remit or knowledge just to build a rapport so staff would treat me with a bit more respect less/snide remarks 
Table 4 Responses from nursing and medical students describing the general atmosphere within the clinical environment (these are direct quotes selected by the author to indicate the spread and issues involved)

\section{'If yes, can you provide brief details?'}

M2 Staff talking negatively about other staff

M12 Rude staff to students

N31 My last placement: all staff spoke about each other behind their backs. There was a lot of rivalry and lots of members of staff would not work with other members

N32 Between the doctors and nurses there is sometimes conflict when patients have deteriorated

\section{'What did you do in this situation?'}

Nothing

Came in less

I have informed my mentor and the sister that it is not a pleasant environment to work or learn in

Listened as a learning experience and stayed out the way
Furthermore, nursing students' reports describe a wide range of 'perpetrators', including fellow nurses, healthcare assistants and clinicians as well as the involvement of patients (both in the role of victim and source). Meanwhile, medical students experiences appear primarily restricted to their own profession; they often describe being belittled by the clinicians who teach them and they recount observing trainees being bullied by senior doctors. This finding mirrors the GMC's NTS survey, which found that in $44 \%$ of cases, trainees identified their consultant (or General Practice (GP) trainer) as the source. ${ }^{2}$

Studies of the socialisation into the culture of medicine or students' enculturation into the profession provide valuable insights ${ }^{18} 19$ and highlight the remarkable stability of medical education, where salient characteristics remain unchanged over long periods. ${ }^{20} 21$ Bullying and teaching by humiliation and strictly hierarchical structures (in some specialties) appear to be among those. ${ }^{22}$ Sinclair attributes this stability to medicine's insularity and continuing autonomy. ${ }^{21}$

\section{Students' responses and consequences}

There are also important differences in students' reported reactions following exposure to bullying and harassment. Most medical respondents reported doing 'nothing' and/or went on to avoid the person they identified as the source of bullying. By contrast, nursing student respondents most frequently reported de-briefing with their dedicated clinical teacher or other nurses. In addition, several nursing respondents' reports described challenging those involved and/or making a report.

Frank $^{7}$ reported that a third of affected medical trainees did not deem the incidences sufficiently serious to warrant action. Given that medical respondents identified consultants as the source of the bullying it seems likely that this failure to report is related to fear and disempowerment in a hierarchical organisational culture. ${ }^{9-11}$ Seabrook questions the potential function of students' acceptance of intimidation: she concludes that it is a means of demonstrating one's successful socialisation into the norms and values of medicine. ${ }^{10}$ Our survey adds to these insights by suggesting that medical students might be missing out on reflecting on their experiences and learning from them in the ways that nursing students appear to do already. Haglund et al argue that medical students' personal growth can and does follow traumatic experiences during the first clinical year-crucially, however, it relies on discussing events with members of the clinical team. ${ }^{23}$

In our survey medical student respondents reported 'feeling bad' or finding it 'harder to concentrate' as a consequence of their exposure to bullying and harassment. The GMC's report shows that those trainees who experience bullying and undermining are less satisfied with their training. ${ }^{2}$ A US survey found a significant association between poor mental health and low career

Table 5 Responses from nursing and medical students that mention patients either as a source or participant to bullying and harassment (these are direct quotes selected by the author to indicate the spread and issues involved)

\section{'If yes, can you provide brief details?'}

M5 A fellow colleague was touched inappropriately by a patient whilst taking blood

N10 Staff to patient

N39 From a patient who presented with aggressive and violent behaviour due to current mental state

\section{'What did you do in this situation?'}

Stopped the patient from what he was doing, spoke to him about it and what he did. Then made sure my colleague was OK and talked to her about it

Report the staff member

[Sought] supervision with mentor when required, tried to develop skills to help in these situations 
satisfaction among those who had been harassed or belittled. ${ }^{7}$ Haglund et $a l^{23}$ suggest that mistreatment by superiors adversely affects the well-being of students and may impact negatively on students' empathy in the longer term. Wood cites associations with alcohol misuse, depression and suicidal intent. ${ }^{24}$

\section{CONCLUSION}

Despite bullying and harassment clearly being an issue affecting healthcare organisations across the UK-as exemplified by the NHS Bullying Survey ${ }^{25}$ and a 2013 report funded by the National Institute for Health Research $^{26}{ }^{27}$ - until now no quantitative data has been published by any of the UK medical schools. While it seems unlikely that medical schools are unaware of the issues, their hesitation to investigate (or publish) can be questioned. In the US A, the University of California Los Angeles (UCLA) medical school has taken a bold stand in openly discussing not only their quantitative results from surveys of bullying, but also their difficulties in and strategies for addressing student mistreatment. ${ }^{28}$

Within the UK context-despite a series of wellmeaning editorials, ${ }^{24} 29{ }^{30}$-until quite recently the debate on bullying and harassment had remained somewhat stagnant. Following the publication of the Francis Review, ${ }^{31}$ which underlined the connection with poor patient outcomes and in view of the GMC's concern over trainee mistreatment, ${ }^{2}$ this may be an ideal moment to recognise that bullying and harassment occur already in the first clinical year of medical school. Box 2 (below) outlines suggestions for UK education bodies on how the issues raised might be addressed nationally.

Our nursing data suggests a higher degree of exposure to bullying and harassment within that profession, but as noted, this may reflect a greater willingness to report and address the issues. Back in 2002 the Royal College of Nursing published a guide for nursing students on how to deal with bullying and harassment. ${ }^{33}$ The comparison

Box 2 Suggestions to the General Medical Council (GMC) and Medical School's Council on how to address undergraduates' exposure to bullying and harassment.

- For the GMC to share National Training Survey (NTS) findings requiring follow-up also with local medical schools. This may happen already, but at present the GMC commits only to involvement of the deaneries. ${ }^{2}$

- For the GMC to introduce an NTS-equivalent survey for undergraduate students. Sector-wide statistics would enable comparisons between postgraduate (PG) and undergraduate (UG) data and across different areas and address concerns about single centre surveys and sample size. ${ }^{24}$ An annual survey would also allow for the observation of trends.

- For the Medical School's Council to review guidelines for admissions to medicine and to suggest consideration of applicants' resilience. ${ }^{23} 32$ with medicine highlights that (some) nursing students are already willing (and enabled) to confront such challenges. Since the Francis Review was much more prescriptive about nurse training than medical education, further developments are to be expected. ${ }^{31}$ Regardless, the above recommendations may still be of interest to the Nursing and Midwifery Council.

Given that bullying and harassment have been documented within medical training for almost 30 years, ${ }^{30}$ these issues are unlikely to disappear quickly. Moreover, as noted in the discussion, the literature on socialisation into the culture of medicine continues to be pertinent. Recognising bullying and harassment is a crucial issue for students-even those in their first year of full-time placements-as well as institutions, that is, medical schools, hospitals and the Colleges. At this point in time, as the GMC is becoming more assertive in protecting doctors in training, the question remains whether it will respond also to protect its most junior members from mistreatment and toxic role-modelling.

Acknowledgements Following discussion with the editor and reviewers, it was decided to prioritise anonymity for the institutions and collaborators involved in the larger project on which this paper is based. The larger project was conducted at two English institutions, though only one data set is reported here. Financially, this work was supported by the Higher Education Funding Council for England (through the Higher Education Academy).

The questionnaire for the survey was originally designed by members of the project team. This was followed by additional separate development, which effectively resulted in two surveys. Whilst the survey ran, crucial input was provided by members of the administrative teams at the research sites who assisted in the recruitment of respondents.

This manuscript benefitted greatly from the careful reading and comments provided by colleagues at Southampton: Faith Hill, Brenda Johnston, Kathy Kendall and especially, Catherine Pope. I am also grateful to the reviewers and editor of BMJ Open for their helpful advice.

Funding This work was supported by the Higher Education Funding Council for England (through the Higher Education Academy). In the interest of anonymity, it was agreed with the funder not to supply further details.

Competing interests None.

Ethics approval University of Southampton, Faculty of Medicine Ethics Committee.

Provenance and peer review Not commissioned; externally peer reviewed.

Data sharing statement No additional data are available.

Open Access This is an Open Access article distributed in accordance with the Creative Commons Attribution Non Commercial (CC BY-NC 4.0) license, which permits others to distribute, remix, adapt, build upon this work noncommercially, and license their derivative works on different terms, provided the original work is properly cited and the use is non-commercial. See: http:// creativecommons.org/licenses/by-nc/4.0/

\section{REFERENCES}

1. Anon. Key findings: national training survey 2012. General Medical Council; Report, 2012. http://www.gmc-uk.org/education/national_ summary_reports.asp (accessed Feb 2014).

2. Anon. National training survey 2013: undermining (Autumn Report). General Medical Council; Report, 2013. http://www.gmc-uk.org/ education/surveys.asp (accessed Feb 2014).

3. Anon. Joint Statement. Royal College of Obstetricians and Gynaecologists (RCOG) and Royal College of Midwives (RCM). 2013. http://www.rcog.org.uk/news/rcogrcm-press-announcementroyal-colleges-jointly-address-issue-undermining-and-bullying-workpl (accessed Nov 2013) 
4. Anon. Undermining and bullying consensus statement. Association of Surgeons in Training (ASiT), 2013. http://www.asit.org/resources/ articles/undermining\&bullying (accessed Nov 2013).

5. Quine L. Workplace bullying in junior doctors: questionnaire survey. BMJ 2002;324:878-9.

6. Paice $\mathrm{E}$, Aitken $\mathrm{M}$, Houghton $\mathrm{A}$, et al. Bullying among doctors in training: cross sectional questionnaire survey. BMJ 2004;329:658.

7. Frank E, Carrera JS, Stratton T, et al. Experiences of belittlement and harassment and their correlates among medical students in the United States: longitudinal survey. BMJ 2006;333:682.

8. Lempp $H$, Seale $C$. The hidden curriculum in undergraduate medical education: qualitative study of medical students' perceptions of teaching. BMJ 2004;329:770-3.

9. Seabrook MA. Clinical students' initial reports of the educational climate in a single medical school. Med Educ 2004;38:659-69.

10. Seabrook MA. Intimidation in medical education: students' and teachers' perspectives. Stud Higher Educ 2004;29:59-74.

11. Hafferty FW. Beyond curriculum reform: confronting medicine's hidden curriculum. Acad Med 1998;73:403-7.

12. Rees CE, Monrouxe LV. "A Morning since eight of just Pure Grill": a multi-school qualitative study of student abuse. Acad Med 2011;86:1374-82.

13. Rees CE, Monrouxe LV, McDonald LA. Narrative, emotion and action: analysing "most memorable" professionalism dilemmas. Med Educ 2013;47:80-96.

14. Schuchert MK. The relationship between verbal abuse of medical students and their confidence in their clinical abilities. Acad Med 1998;73:907-9.

15. Musselman LJ, MacRae HM, Reznick RK, et al. 'You learn better under the gun': intimidation and harassment in surgical education. Med Educ 2005;39:926-34.

16. Gordon J, Markham P, Lipworth W, et al. The dual nature of medical enculturation in postgraduate medical training and practice. Med Educ 2012;46:894-902.

17. Health Policy \& Economic Research Unit. Bullying and harassment of doctors in the workplace. British Medical Association; Report, 2006.

18. Atkinson P. The clinical experience: the construction and reconstruction of medical reality. Farnborough: Gower, 1981.

19. Hafferty FW. Cadaver stories and the emotional socialization of medical students. J Health Soc Beh 1988;29:344-56.
20. Bloom SW. The medical school as a social organisation: the source of resistance to change. Med Educ 1989;23:228-41.

21. Sinclair S. Making doctors. An institutional apprenticeship. Oxford: Berg, 1997.

22. Cleland J, Johnston P. Enculturation to medicine: power for teachers or empowering learners? Med Ed 2012;46:830-7.

23. Haglund MEM, aan het Rot M, Cooper NS, et al. Resilience in the third year of medical school: a prospective study of the associations between stressful events occurring during clinical rotations and student well-being. Acad Med 2009;84:258-68.

24. Wood DF. Bullying and harassment in medical schools. BMJ 2006;333:664.

25. National NHS Staff Survey. http://www.nhsstaffsurveys.com/Page/ 1006/Latest-Results/2013-Results/ (accessed Apr 2014).

26. Illing JC, Carter M, Thompson NJ, et al. Evidence synthesis on the occurrence, causes, consequences, prevention and management of bullying and harassing behaviours to inform decision making in the NHS. Final report. NIHR Service Delivery and Organisation programme, 2013. http://www.nets.nihr.ac.uk/projects/hsdr/10101201 (accessed Feb 2014).

27. Carter $\mathrm{M}$, Thompson $\mathrm{N}$, Crampton $\mathrm{P}$, et al. Workplace bullying in the UK NHS: a questionnaire and interview study on prevalence, impact and barriers to reporting. BMJ Open 2013;3:e002628.

28. Fried JM, Vermillion M, Parker $\mathrm{NH}$, et al. Eradicating medical student mistreatment: a longitudinal study of one institution's efforts. Acad Med 2012;87:1191-8

29. Pope C, Coldicott Y. No-one forgets a bad teacher. Med Educ 2002;36:5-6.

30. Neville AJ. In the age of professionalism, student harassment is alive and well. Med Educ 2008;42:447-8.

31. Francis R. Report of the Mid Staffordshire NHS Foundation Trust Public Inquiry. The Francis Report 2012. Report number: HC 947. London: The Stationery Office, 2012. http://www.midstaffspublicinquiry.com/ report (accessed Jan 2014).

32. Howe A, Smajdor A, Stöckl A. Towards an understanding of resilience and its relevance to medical training. Med Educ 2012;46:349-56.

33. Anon. Dealing with Bullying and Harassment-a guide for nursing students. Royal College of Nursing, 2002. (reprinted 2005) http:// www.rcn.org.uk/_data/assets/pdf_file/0011/78518/001497.pdf (accessed Feb 2014). 